\title{
Publication E. Viscoelasticity and High Buckling Stress of Dense Carbon Nanotube Brushes
}

Siddhartha Pathak, ${ }^{\text {a,b }}$ Z. Goknur Cambaz, ${ }^{\text {a }}$ Surya R. Kalidindi, ${ }^{a}$ J. Gregory Swadener, ${ }^{c}$ Yury Gogotsi $^{\text {a }}$

${ }^{a}$ Department of Materials Science and Engineering and A.J. Drexel Nanotechnology Institute, Drexel University, Philadelphia, PA 19104, USA

${ }^{\mathrm{b}}$ Laboratory for Mechanics of Materials and Nanostructures, EMPA - Swiss Federal Lab for Materials Testing and Research, Thun, CH-3602, Switzerland

${ }^{c}$ Center for Integrated Nanotechnologies, Los Alamos National Laboratory, Los Alamos, NM 87545, USA. Currently at: Engineering Systems \& Management, Aston University, Aston Triangle, Birmingham B4 7ET, UK

Carbon. 47, 2009, pg 1969-76.

\section{Abstract}

We report on the mechanical behavior of a dense brush of small-diameter (1-3 nm) noncatalytic multiwall (2-4 walls) carbon nanotubes (CNTs), with $\sim 10$ times higher density than CNT brushes produced by other methods. Under compression with spherical indenters of different radii, these highly dense CNT brushes exhibit a higher modulus ( 17-20 GPa) and orders of magnitude higher resistance to buckling than vapor phase deposited CNT brushes or carbon walls. We also demonstrate the viscoelastic behavior, caused by the increased influence of the van der Waals' forces in these highly dense CNT brushes, showing their promise for energy-absorbing coatings. 


\section{Introduction}

Layers of vertically aligned carbon nanotubes (CNTs), known as CNT brushes, forests or arrays, have been suggested for applications in superhydrophobic, compliant and energyabsorbing coatings $[1,2]$. While individual CNTs have been announced as the strongest material known [3] and have shown extremely high strength and Young's modulus in tensile tests on individual tubes [4], much less is known about the CNT brushes in terms of their mechanical behavior. Moreover, the low density of the reported CNT brushes[1, 5, 6] also poses a serious obstacle in obtaining significant compression strength/modulus needed for their energyabsorbing applications.

Instrumented indentation has been the most common method for studying mechanical properties of films and coatings, however, only a handful of studies have been conducted on CNT brushes. The extremely high aspect ratio of the CNTs makes them highly susceptible to buckling under compression [6], which is generally believed to be the reason for the significantly lower values of stiffness, ranging anywhere from 0.25 [7] to 50 [1] to 800 [8] MPa, reported during nanoindentation compression experiments on CNTs, as compared to exceptionally high values computed from theory [9] and tensile tests ( 1-5 TPa [3]). At the same time, the ability to elastically sustain loads at large deflection angles has earmarked the CNTs as uniquely tough materials for energy-absorbing applications [10]. Buckling and post-buckling analysis of CNTs under a variety of loading conditions such as axial compression, bending, torsion and external pressure have been studied by simulation [9, 11-14]. Experimentally, large diameter ( 100 nm) CNTs produced in alumina templates with no catalyst added were tested using a sharp (Berkovich) and a spherical indenter [5], as well as a flat punch [6] and a low buckling load $(\sim 2.0-2.5 \mu \mathrm{N})$ was measured. Very low buckling loads and high compliance of nanotubes were also observed for long CVD tubes in compression tests [8]. The modulus measured in compression was well below $1 \mathrm{GPa}$, in contrast to the tens to hundreds GPa in tension, with even lower values reported for CNT turf [15]. A compressive modulus of $0.25 \mathrm{MPa}$ was measured for 20-30 nm diameter CNT brushes and was found to be independent on the array height [7]. Deformation during compression for CVD CNT films has been shown to be reversible in both, normal [1] and radial [16] directions. Sidewall chemical modification has been shown to affect interaction of the CNTs with the indenter (friction) [17]. Indentation studies have also been done 
on CNT-polymer composites [18], but those provide little information about properties of nanotubes inside the composite.

Indentations with an atomic force microscope (AFM) tip have also been used to study 50$100 \mathrm{~nm}$ diameter tubes vertically aligned in CNT films grown by catalytic chemical vapor deposition (CVD) and a very high bending modulus was reported [19]. Low buckling loads ( 30 $\mathrm{nN}$ ) and mechanical instabilities, including viscoelasticity and negative stiffness regimes (where the axial force decreases with increasing compression), have been reported in individual multiwalled CNTs (MWCNTs) for a wide range of aspect ratios. The negative stiffness behavior of the CNTs is highly promising as a possible route to designing composite systems with enhanced overall stiffness and damping [20, 21]. Similar negative stiffness regimes have also been demonstrated for single-walled CNTs (SWCNTs) using molecular dynamic simulations [9, $14]$

It is important to note that, while small-diameter SWCNTs show much higher tensile strength and modulus compared to large-diameter MWCNTs [22], no indentation studies have been performed on double-wall CNTs or brushes of SWCNTs. One of the reasons may be the extremely low density of SWCNT brush ( $>90 \%$ porosity). Super-compressible MWCNTs reported by Cao et al. [1] had porosity of about $87 \%$. It is very difficult to expect any significant compression strength/modulus from such a porous body. In addition, since tubes in CVD arrays are located at distances of at least a tube diameter (often as much as 100-200 $\mathrm{nm}[6,8]$ ) from each other, the interactions between the tubes in an array are weak and a low energy dissipation is expected [13]. Similarly the negative stiffness of CNTs has only been demonstrated for individual MWCNTs [20, 21], whereas any practical application of these materials in composite systems is likely to involve a large number ( thousands to millions) of CNTs in close proximity to each other.

Although lower density and wider spaced CNT brushes have been indented with flat punch [6,7] and sharper Berkovich [5] indenters, each of these indenters have their own disadvantages. While maintaining parallel contact between the indenter and the sample is a major concern for the flat punch indenter [5], the sharper contact in Berkovich indentation can cause the CNTs to bend away from the indenter [19]. On the other hand, the smoother stress fields in spherical indentation, as compared to sharper indenters [23], allows indentation stress-strain curves to be extracted from the raw load-displacement data [24-26], which in turn enables one to 
follow the entire evolution of the mechanical response in the CNT array, from initial elasticity to the initiation of buckling to post-buckling behavior at finite plastic strains. Because of these advantages, spherical nanoindentation with 3 different indenter tip sizes (1, 5 and $13.5 \mu \mathrm{m}$ radii) was chosen to analyze the buckling response of the CNT brushes in this work. The higher density of these brushes, which makes them ideal for indentation testing, is evident in the scanning electron micrograph (SEM) image in Fig. 1a of the CNT brush grown at $1700{ }^{\circ} \mathrm{C}$, where no apparent porosity is visible.
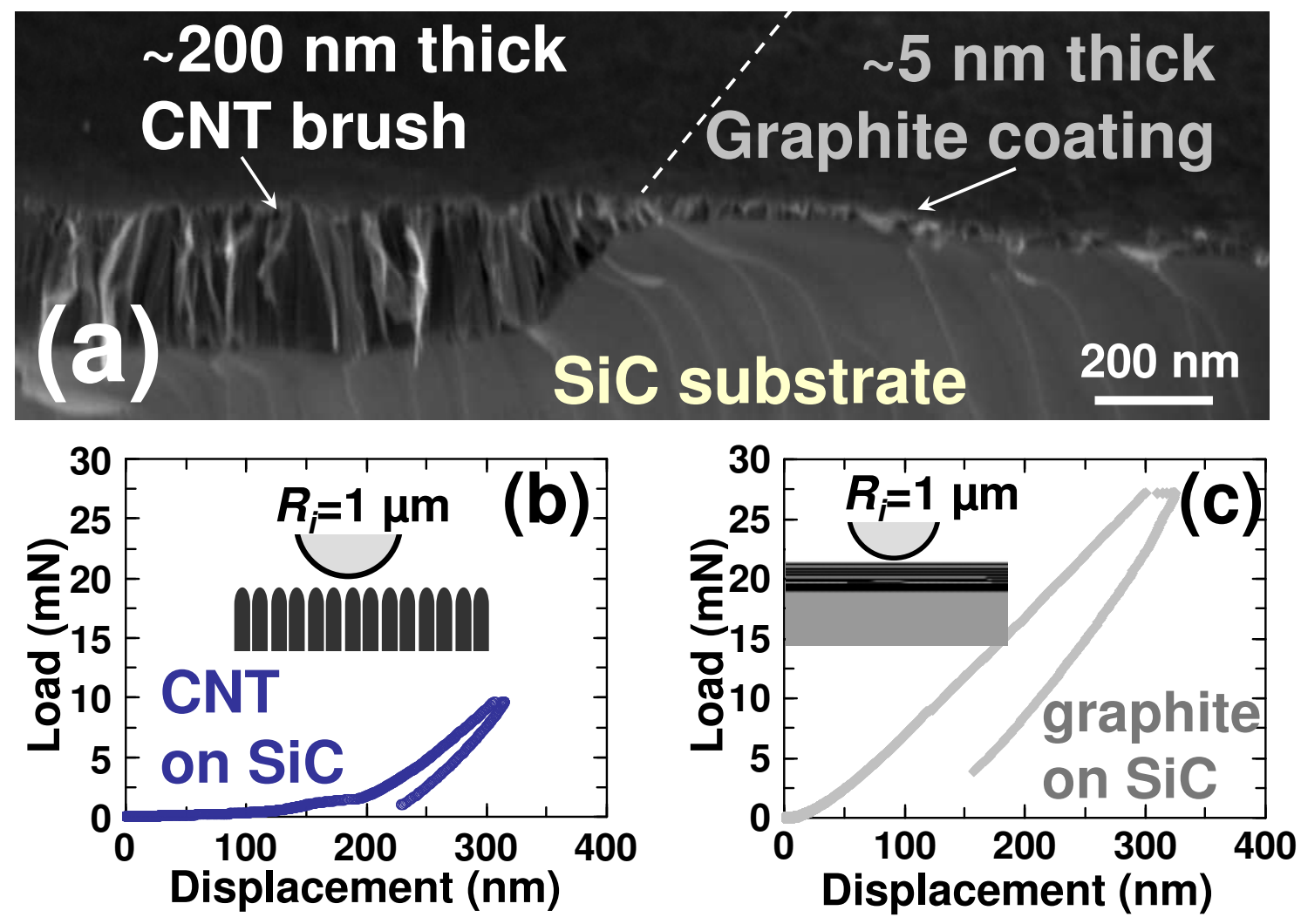

Figure 1. Indentation scheme used to analyze the mechanical properties of the CNT brushes. (a) SEM micrograph showing the CNT brush - graphite interface. The $\mathrm{SiC}$ wafers were treated at $1700^{\circ} \mathrm{C}$. (b) and (c) show a comparison of spherical indentation (indenter radius, $R_{i}=1 \mu \mathrm{m}$ ) load-displacement responses between the CNT brush and the graphite coating up to an indentation depth of $300 \mathrm{~nm}$.

The lower density of the CVD CNT brushes [1, 6, 8] also makes them unsuitable for measuring their viscoelastic properties. Although nanoindentation studies have often been used in characterizing the viscoelastic behavior in solids [27, 28], their utility in measuring the viscoelasticity of CNTs has been limited to studies of CNT-polymer composites [29-31]. The 
dense CNT brushes produced in this work thus provide a unique opportunity to address the time dependent mechanical behavior of the CNT brush assembly.

In this paper, we report on the mechanical behavior under contact loading of dense CNT brushes ( 10 times higher density than CNT brushes produced by other methods) produced by vacuum decomposition of SiC. Our results demonstrate a significantly higher loading modulus and buckling resistance in these dense CNT brushes. The CNT brushes are shown to exhibit negative stiffness in their post-buckled regime - a behavior previously noted only in individual CNTs. The close proximity of the CNTs in these highly dense brushes also results in an increased influence of van der Waals' forces between the tubes, which is evident in their viscoelastic behavior during indentation.

\section{Experimental}

\subsection{Sample preparation}

In this work, highly dense CNT brushes were produced by high temperature vacuum decomposition of $6 \mathrm{H} \mathrm{SiC}$ single crystals. The $6 \mathrm{H}-\mathrm{SiC}$ single crystal wafers, $0.37 \mathrm{~mm}$ in thickness with epi-ready polished (0001) Si face and optical polished (0001) C face on axis without any dopants (resistivity $>10^{5} \mathrm{Ohm} \cdot \mathrm{cm}$ ), were obtained from Intrinsic Semiconductor Corporation. It has been shown [32] that high temperature decomposition of $\mathrm{SiC}$ by the reactions

$\mathrm{SiC} \rightarrow \mathrm{Si}(\mathrm{g})+\mathrm{C}$

$\mathrm{SiC}+1 / 2 \mathrm{O}_{2}(\mathrm{~g}) \rightarrow \mathrm{SiO}(\mathrm{g})+\mathrm{C}$

leads to the formation of CNTs growing normal to the carbon terminated (0001) C-face of hexagonal $\mathrm{SiC}$ with primarily zigzag chirality [33] and graphite growth on the Si terminated (0001) Si-face. These carbide-derived carbon (CDC) nanotube brushes have been shown to have a density close to $0.95 \mathrm{~g} / \mathrm{cm}^{3}$ [34], which is significantly (10 times or more) higher than in catalytic CVD growth of any kind of nanotubes (see also Fig. 1a). This higher density is generally thought be due to a conformal transformation of SiC into carbon. These dense CDC CNT brushes consist of small-diameter (1-3 nm outer diameter, 1-4 walled) non-catalytic CNTs with double walled CNTs being the most common, as determined from transmission electron microscopy (TEM), and a strong RBM mode in Raman spectra [34]. Assuming an average outer diameter of $3 \mathrm{~nm}$ and $0.35 \mathrm{~nm}$ as the inter-tube distance, this would correspond to an aerial 
density of $\sim 100,000$ tubes per $\mu \mathrm{m}^{-2}$ for a hexagonal arrangement of CNTs, and $\sim 89,000$ tubes per $\mu \mathrm{m}^{-2}$ for a square arrangement. The actual aerial density of the CNTs packed randomly in a dense brush is likely to be somewhere in between.

Three different sets of samples were used in this study. Graphite or CNT structure, and thickness of carbon coating on $\mathrm{SiC}$ were controlled by changing the synthesis temperature as described by Cambaz et al. [34]. The first set of samples (Figs. 1 and 2) were produced by heating the $\mathrm{SiC}$ wafers to $1700{ }^{\circ} \mathrm{C}$ for $4 \mathrm{hrs}$ in a Solar Atmospheres (US) vacuum furnace with an electric resistance carbon heater. The heating rate was $5^{\circ} \mathrm{C} \min ^{-1}$. For these samples the $\mathrm{SiC}$ wafers were patterned by sputtering carbon on the C-face. Sputtering of carbon formed a diffusion barrier and largely suppressed the growth of CNT on the C-face, allowing tubes to grow only on the coating-free area. $\sim 200 \mathrm{~nm}$ thick CNT brush patterns were grown by this technique. Significantly thicker $(1.2-1.4 \mu \mathrm{m}$ thickness $)$ CNT brushes were grown in the second set of samples (shown in Figs. 3 and 4) by heating the $\mathrm{SiC}$ wafers to $1800{ }^{\circ} \mathrm{C}$ for $4 \mathrm{hrs}$. The final set of samples (Fig. 4, Test C) consisted of a dense $\sim 3 \mu \mathrm{m}$ thick CNT/carbon wall layer grown by heating the $\mathrm{SiC}$ wafers to $1900{ }^{\circ} \mathrm{C}$ for $4 \mathrm{hrs}$. All samples were investigated by using fieldemission SEM (Supra 50VP, Zeiss, Germany). For these investigations, samples were fractured after vacuum decomposition to study both the cross-section and the surface of the coatings.

\subsection{Indentation Stress-Strain Curves}

The MTS NanoIndenter $\mathrm{XP}^{\circledR}$ (Nano Instruments, MTS Systems Corporation, USA) was used for the spherical nanoindentation studies. Standard protocols prescribed in the MTS machine manual were used to determine the machine frame stiffness, the harmonic frame stiffness and other calibrations. In order to probe different material volumes in the CNT brushes, three diamond indenter tips of increasing tip radii $(1,5$ and $13.5 \mu \mathrm{m})$ were used in this work. The choice of these three indenter tip sizes results in varying indentation zone sizes at buckling, ranging from values much smaller than the film thickness to the values larger than the film thickness.

The tests were carried out under closed loop displacement control using the continuous stiffness measurement (CSM) attachment. Several (15-20) tests were conducted to ascertain repeatability. Similar tests, using the same indenter tips, were carried out in aluminum, fused silica and tungsten with known values of Young's moduli, and these were used to validate the 
procedures described below. Note that the CSM applies a very small sinusoidally varying signal ( $2 \mathrm{~nm}$ oscillations) on top of the DC force signal driving the motion of the indenter. For a material with a low modulus-to-hardness ratio such as the CNT brushes (modulus-to-hardness ratio $\sim 45)$ the amplitude of these essentially elastic oscillations is unlikely to affect the deformation process [35]. Further, since the CSM signal was used in this work to calculate the contact radius during indentation (see Eq. (4) below), these values are also not expected to be affected by concerns of edge or substrate effects raised in a few recent reports [36]. Note also that the contact stiffness of the CNT brushes is significantly smaller than the CSM machine stiffness - for example, the elastic stiffness (as measured by the CSM) at buckling for the 1.2-1.4 $\mu \mathrm{m}$ thick CNT brush using the larger $13.5 \mu \mathrm{m}$ indenter is $\sim 70,000 \mathrm{~N} / \mathrm{m}$ while the machine stiffness of the CSM is around $8 \times 10^{6} \mathrm{~N} / \mathrm{m}$, and as such, it is unlikely to affect the measurements for the indenter sizes used in this study.

Indentation stress-strain curves were extracted from the raw load-displacement-CSM data following the technique described in our recent papers [24, 25]. In brief, the extraction of reliable indentation stress-strain curves from the measured load displacement data is essentially a twostep process. The first step in this process is an accurate estimation of the point of effective initial contact in the given data set, i.e. a clear identification of a zero-point that makes the measurements in the initial elastic loading segment consistent with the predictions of Hertz's theory [37, 38]. For spherical nanoindentation this relationship was expressed as [24, 25]

$$
S=\frac{3 P}{2 h_{e}}=\frac{3\left(\tilde{P}-P^{*}\right)}{2\left(\tilde{h}_{e}-h^{*}\right)},
$$

where $\tilde{P}, \tilde{h}_{e}$, and $S$ are the measured load signal, the measured displacement signal, and the CSM signal in the initial elastic loading segment from the machine, and $P^{*}$ and $h^{*}$ denote the values of the load and displacement signals at the point of effective initial contact. A linear regression analysis was used to establish the point of effective initial contact ( $P^{*}$ and $\left.h^{*}\right)$ in the indentation experiment.

In the second step of the data analysis method for the extraction of the indentation stressstrain curves, Hertz's theory $[37,38]$ was recast in the following set of equations for frictionless, elastic, spherical indentation (see also [24, 25]): 


$$
\begin{aligned}
& \sigma_{\text {ind }}=E_{\text {eff }} \varepsilon_{\text {ind }}, \quad \sigma_{\text {ind }}=\frac{P}{\pi a^{2}}, \quad \varepsilon_{\text {ind }}=\frac{4}{3 \pi} \frac{h_{e}}{a} \approx \frac{h_{e}}{2.4 a}, \\
& a=\frac{S}{2 E_{\text {eff }}}, \quad \frac{1}{E_{\text {eff }}}=\frac{1-v_{s}^{2}}{E_{s}}+\frac{1-v_{i}^{2}}{E_{i}}, \quad \frac{1}{R_{\text {eff }}}=\frac{1}{R_{i}}+\frac{1}{R_{s}},
\end{aligned}
$$

where $\sigma_{\text {ind }}$ and $\varepsilon_{\text {ind }}$ are the indentation stress and the indentation strain, $a$ is the radius of the contact boundary at the indentation load $P, h_{e}$ is the elastic indentation depth, $S$ is the elastic stiffness described earlier, $R_{\text {eff }}$ and $E_{\text {eff }}$ are the effective radius and the effective stiffness of the indenter and the specimen system, $v$ and $E$ are the Poisson's ratio and the Young's modulus, and the subscripts $s$ and $i$ refer to the specimen and the indenter, respectively. When compared to other currently used data analysis methods $[39,40]$, these indentation stress-strain curves have been demonstrated to provide much more reliable estimates for the elastic moduli measured in loading and unloading segments [24-26], and the changes in the indentation yield points [41]. These methods have also been used for identifying and explaining several of the surface preparation artifacts typically encountered in nanoindentation measurements [42].

\subsection{Visco-elastic Nanoindentation}

The micro-scale dynamics properties of the CNT brushes were analyzed using the nanoDMA (Dynamic Mechanical Analysis) mode of the Hysitron Triboscope ${ }^{\circledR}$ (Hysitron Inc., Minneapolis, MN, USA) with a $1 \mu \mathrm{m}$ radius spherical fluid tip. The nano-DMA technique takes into account the elastic as well as the viscous contribution of the test material, which allows the calculation of $\tan \delta$. In the frequency mode of the nano-DMA software the applied load is specified, which fixes the indentation depth, and the probe is oscillated across a range of frequencies. The frequency dependent dynamic response of the material can be ascertained using the following equation

$$
\tan \delta=\frac{E^{\prime \prime}}{E^{\prime}}=\frac{\omega C_{S}}{k_{S}}
$$

where $E^{\prime}$ and $E^{\prime \prime}$ denote the storage and loss moduli, $\omega$ is the frequency of the applied force and $k_{S}$ and $C_{S}$ are the sample stiffness and damping coefficients respectively. Details of the model can be found in Ref. [27]. The indenter was held for 2 mins at the applied load in order to dissipate any creep effects before starting the probe oscillations. Standard protocols prescribed in the machine manual for the Triboscope ${ }^{\circledR}$ and the nano-DMA were used to determine the machine 
frame stiffness and other calibrations. Similar tests were done on polycarbonate with known values of $\tan \delta$ to validate the accuracy of the method. The noise level in the machine, determined by conducting frequency sweeps in non-viscoelastic materials such as aluminum and tungsten, was found to be around $0.03 \tan \delta$.

\section{Results and discussion}

\subsection{Buckling of CNTs}

The SEM micrograph of the CNT brush - graphite interface, produced by heating the SiC wafers to $1700{ }^{\circ} \mathrm{C}$ for $4 \mathrm{hrs}$, is shown in Fig. 1a. Figs. 1b and 1c show a comparison of the indentation load-displacement responses, measured with a $1 \mu \mathrm{m}$ spherical indenter, between a thin CNT brush (thickness $\sim 200 \mathrm{~nm}$ ) and a thinner (thickness $\sim 5 \mathrm{~nm}$ ) graphite coating on a patterned single-crystal SiC substrate. As seen from Fig. 1b, after about $200 \mathrm{~nm}$ of penetration into the CNT brush, the indenter experiences the SiC substrate, which is marked by a sharp rise in the load-displacement response. On the other hand, the corresponding curve for indentation on the thinner graphite coating (Fig. 1c) shows an almost immediate influence of the SiC substrate. These two distinctly different indentation behaviors allow one to determine the location of the CNT brushes using only the mechanical response. The lower hardness of the CNT brush compared to the graphite film on the $\mathrm{SiC}$ substrate is also evident from these figures.

It is interesting to note that the indentation response of the CNT brush with the spherical indenter (see the expanded view of the CNT indentation curve in Fig. 2a) is qualitatively similar to the one recorded with a flat punch indenter for 25-nm MWCNTs partially released from an alumina membrane [6], as well as to the axial compression of MWCNT (diameter $30 \mathrm{~nm}$, aspect ratio 80-220) attached to an AFM tip [20]. As in both these cases, three distinct stages are visible during indentation of the CNT brushes: there is an initial linear portion where the indenter elastically compresses the CNT array (see the schematic in Fig. 2b), followed by the initiation of buckling at a critical load (see Fig. 2a inset), and finally a sharp increase in the slope of the curve signaling the influence of the SiC substrate. The associated indentation stress-strain curve, shown in Fig. 2b, depicts these three stages even more clearly while at the same time also allowing one to calculate the elastic modulus and the stress at buckling in the indentation experiment. From this figure, the modulus of these $200 \mathrm{~nm}$ thick CNT brushes was estimated to be $\sim 17 \mathrm{GPa}$ (using Eqs. (3) and (4)) and the critical buckling stress was estimated as $\sim 0.3 \mathrm{GPa}$ at a load of $0.02 \mathrm{mN}$. 

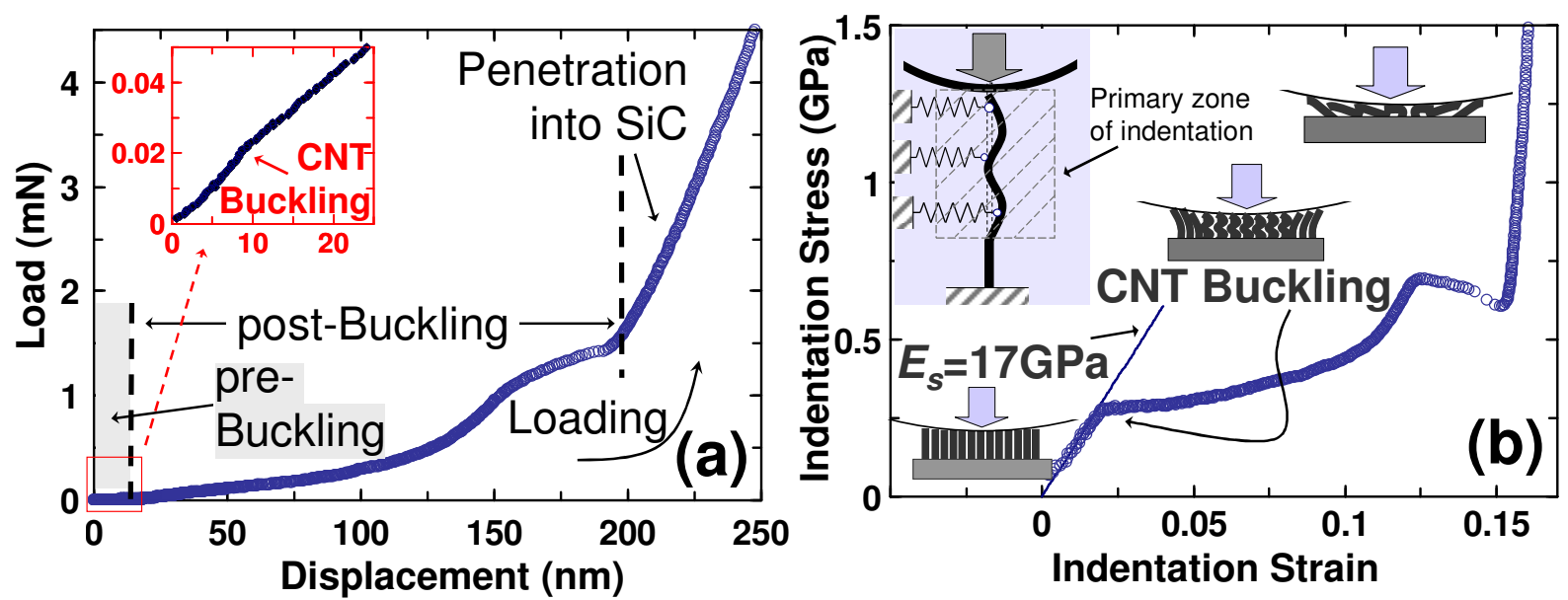

Figure 2. (a) Enlarged view of Fig. $1 \mathrm{~b}$ showing the loading of a $1 \mu \mathrm{m}$ spherical indenter on the $\sim 200 \mathrm{~nm}$ thick CNT brush. Three distinct responses are visible during indentation. (a inset) Enlarged view of the initiation of buckling at a critical load. (b) The corresponding indentation stress-strain curve allows a better representation of these three stages of CNT indentation. (b inset) Schematic illustration of buckling of the CNTs in a dense CNT brush in the indentation zone.
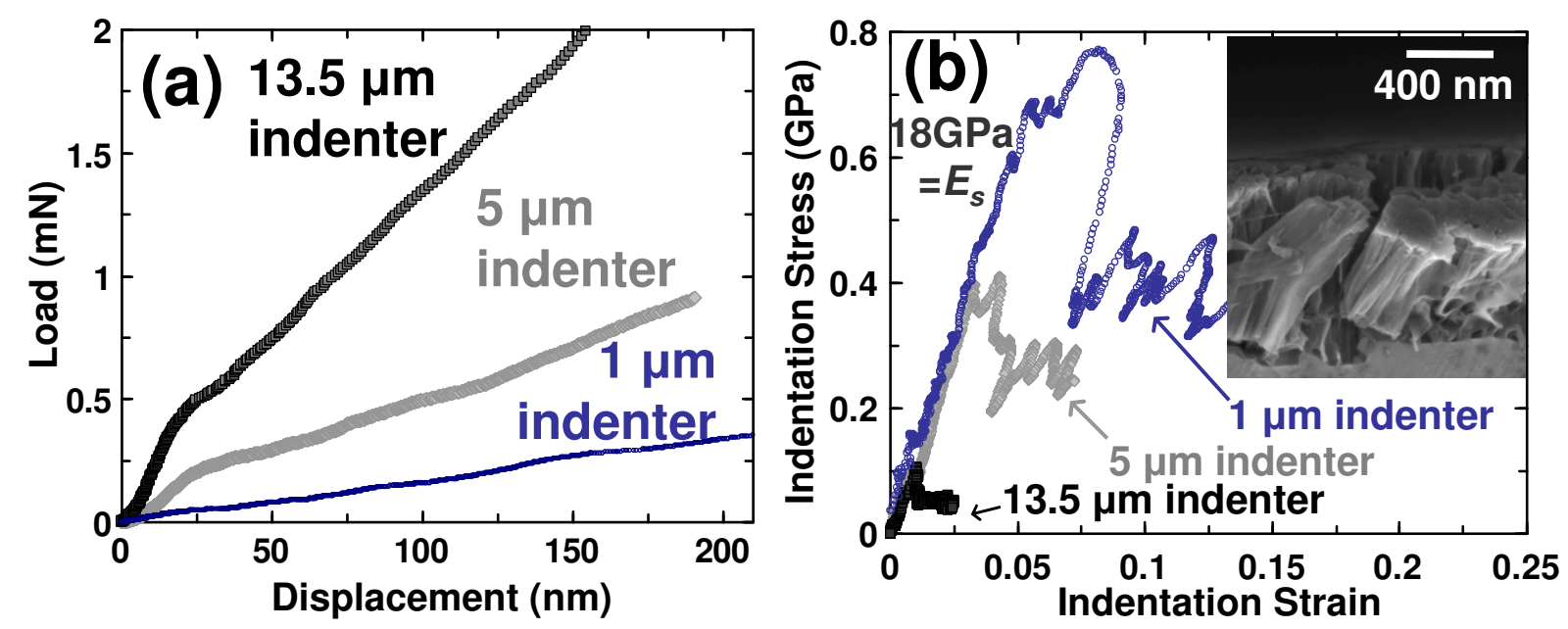

Figure 3. Indentation loading response as a function of indenter radius. The initial elastic behavior followed by the buckling instability is evident from both (a), the load-displacement and (b), the corresponding indentation stress-strain response. Note the lower bucking stress for the larger $13.5 \mu \mathrm{m}$ indenter. (b inset) SEM micrograph of the $1.3 \mu \mathrm{m}$ thick CNT brush grown by decomposition of $\mathrm{SiC}$ at $1800^{\circ} \mathrm{C}$ for 4 hours.

Fig. 3 shows the indentation response on a much thicker CNT brush grown at $1800{ }^{\circ} \mathrm{C}$ (thickness 1.2-1.4 $\mu \mathrm{m}$, see Fig. 3 inset) for 3 different indenter radii. As seen from this figure, the indentation stress-strain response of the CNT brush shows an initial elastic behavior (modulus $\sim 18 \mathrm{GPa}$ ), followed by a sharp drop at a critical stress - a trend that is highly repeatable in 
several experiments conducted across the range of indenter sizes. The good agreement between the indentation stress-strain curves in the initial elastic segments provides additional validation for the data analyses procedures used in this work. The buckling behavior is also discernable as a slight shift in the slope from the associated load-displacement data (Fig. 3a): this transition point is interpreted as the onset of buckling instability in the CNT brush. After buckling, the stress drops significantly for the remainder of the indenter loading. The CSM contact stiffness values after buckling (not shown) indicate these regions of stress drop have negative stiffness values during contact loading of the CNT brushes. Such incremental negative stiffness, where the stress decreases with increasing strain, has been noted before for individual CNTs in modeling $[9,14]$ and in axial compression experiments [20, 21], but not for CNT brushes. The fact that this phenomenon is exhibited even in dense CNT brushes can have significant design implications in devices containing CNTs that take advantage of this behavior.

Table 1. Summarized average and standard deviation (of $\geq 5$ tests) values of indentation buckling stress, contact radius and indentation zone size at buckling for the 3 different indenters used in this work. Indentations were performed on the 1.2-1.4 $\mu$ m thick CNT brush sample shown in Fig. 3.

\begin{tabular}{|l|c|c|c|}
\hline & $\begin{array}{c}\mathbf{1} \boldsymbol{\mu m} \text { radius } \\
\text { indenter }\end{array}$ & $\begin{array}{c}\mathbf{5} \boldsymbol{\mu m} \text { radius } \\
\text { indenter }\end{array}$ & $\begin{array}{c}\mathbf{1 3 . 5} \boldsymbol{\mu m} \text { radius } \\
\text { indenter }\end{array}$ \\
\hline $\begin{array}{l}\text { Indentation buckling } \\
\text { stress }\end{array}$ & $0.59 \pm 0.41 \mathrm{GPa}$ & $0.40 \pm 0.11 \mathrm{GPa}$ & $0.09 \pm 0.02 \mathrm{GPa}$ \\
\hline $\begin{array}{l}\text { Contact radius at } \\
\text { buckling }(a)\end{array}$ & $0.16 \mu \mathrm{m}$ & $0.36 \mu \mathrm{m}$ & $1.49 \mu \mathrm{m}$ \\
\hline $\begin{array}{l}\text { Indentation zone size } \\
\text { at buckling }(\approx 2.4 a)\end{array}$ & $0.39 \mu \mathrm{m}$ & $1.01 \mu \mathrm{m}$ & $3.58 \mu \mathrm{m}$ \\
\hline
\end{tabular}

Another interesting point to note from Fig. 3 is that the values of buckling stresses vary significantly between the three different indenters, where indentation with the smaller $1 \mu \mathrm{m}$ indenter shows the highest buckling stress, followed by the $5 \mu \mathrm{m}$ indenter, while buckling with the largest $13.5 \mu \mathrm{m}$ indenter occurs at a significantly lower indentation stress. This point is further illustrated in Table 1, where the average and standard deviation values (of $\geq 5$ tests) of the indentation buckling stress, and the average values of contact radius $(a)$ and indentation zone size ( 2.4a; see Eq. (4)) at buckling from these tests for the 3 different indenters are presented. The indentation zone for spherical indentation, as described in detail in Refs. [24, 25], is generally approximated as a cylinder of radius $a$ and height $2.4 a$, where the majority of the indentation stresses are confined. Note the significant variation in size of the indentation zone at 
buckling in between the 3 indenters. While the indentation zone for the larger $13.5 \mu \mathrm{m}$ indenter at buckling $(\sim 3.58 \mu \mathrm{m})$ is well beyond the CNT brush thickness $(1.2-1.4 \mu \mathrm{m})$, only a limited thickness of the CNT brush $(\sim 0.39 \mu \mathrm{m})$ is affected by the smaller $1 \mu \mathrm{m}$ indenter at buckling. Unlike uniaxial loading, the stress field during indentation is highly heterogeneous. In fact, beyond a certain depth, the material hardly experiences any significant stress. Thus, with a smaller indenter, there is a much smaller effective buckling length (shorter than the overall CNT film thickness) and hence the material is able to withstand a much higher stress level with the smaller indenter. Note also that for a dense material with low defect density (as in these dense CNT brushes), increase in the indenter probe size also increases the likelihood of encountering a defect in the indentation zone. This is evidenced by large variations observed in the buckling stress values $(0.59 \pm 0.41 \mathrm{GPa})$ for the smaller $1 \mu \mathrm{m}$ indenter. This larger spread stems from the higher sensitivity of the smaller indentation zone to variations in defect density, when different regions of the sample are probed.

These numbers suggest that these CNT brushes have a respectable level of the mechanical properties with the modulus of elasticity 1-2 orders of magnitude higher compared to a CVD CNT turf [15]. The critical buckling loads for the CDC CNT brushes in this work for the larger $13.5 \mu \mathrm{m}$ indenter, which is closest in approximation to flat punch indentation, are found to be in the range of 0.5-0.7 $\mathrm{mN}$. The critical buckling loads for CVD CNT brushes under flat punch indentation have been reported to significantly lower, 2.2-2.6 $\mu \mathrm{N}[5,6]$, in spite of the higher wall thickness (50 nm outer diameter, $40 \mathrm{~nm}$ inner diameter) of the CNTs used in those studies. This difference in orders of magnitude between the two values can be explained by a much higher density of the tubes per unit area which results in considerably higher mechanical properties. Molecular dynamics simulation have shown that a much higher buckling load results from CNT clusters [43] because of van der Waals' interactions between CNTs. Van der Waals' forces act over a range of $<5 \mathrm{~nm}$, so low density CNT turf does not have a cluster strengthening effect. Assuming no carbon loss (as suggested by the conformal coating, Fig. 1b) or addition of carbon from environment, the density [34] of the CNT brush is close to $0.95 \mathrm{~g} / \mathrm{cm}^{3}$, which is significantly higher than for catalytic CVD nanotube brushes [1, 44]. This is of extreme importance for making selective CNT membranes for gas or liquid filtration/separation or CNT coatings for tribological applications. 
The Euler beam theory has generally been used in literature for analyzing the buckling behavior of the CNTs $[1,8]$ with an aspect ratio of greater than 200. According to this theory, the critical buckling load for a hollow pin-ended cylinder in the fundamental mode of buckling is given by [45]

$$
P_{C R}=\frac{\pi^{2} E I}{L^{2}}, I_{x}=\frac{\pi}{4}\left(r_{O}^{4}-r_{i}^{4}\right)
$$

where $P_{C R}$ is the buckling load, $E$ is the elastic modulus, $I$ is the moment of inertia, $L$ is the length of the column, and $r_{o}$ and $r_{i}$ are the outer and inner radii of the hollow cylinder respectively. Using Eq. (6), the critical buckling load for a CNT forest consisting of approximately half a million CNTs (corresponding to the number of CNTs in contact with the largest $13.5 \mu \mathrm{m}$ indenter at buckling, see Table 1) each of average outer and inner diameters of 4 and $3 \mathrm{~nm}$ respectively, column length $1.2 \mu \mathrm{m}$, negligible inter-tube spacing $(0.35 \mathrm{~nm})$, and a modulus value of $1 \mathrm{TPa}$ [4], is calculated to be in the range of $0.03 \mathrm{mN}$. This value is an order of magnitude lower than the values seen from Figs. 2 and 3. Obviously Eq. (6) does not represent buckling of a dense CNT brush.

In a highly dense CNT brush (such as the ones reported in this work) the close proximity of the CNTs, where each tube is separated from its neighbor by a van der Waals' bond length, makes it extremely unlikely for the CNTs to fail independently in the primary buckling mode. Such a dense array of CNTs would then necessitate a close interaction between the CNTs themselves, as well as between the indenter and the CNT - forces which have been generally neglected in literature for wider spaced tubes. In fact, this scenario bears more resemblance to an idealized structure in which a hollow cylinder is supported by an array of springs in the indentation zone along its length (see schematic in Fig. $2 \mathrm{~b}$ inset), which provides it with additional support in withstanding large compressive stresses. Hence these CNTs could be expected to buckle in a more energetically favorable zigzag morphology (multimode buckling) leading to a higher compressive buckling threshold. A detailed quantitative analysis of these support systems is, however, beyond the scope of the present paper.

\subsection{Visco-elastic behavior of CNTs}

Repeated load-unload cycles on these CNT brushes result in two distinct indentation responses from the nanotubes depending on the maximum indentation load used, as shown in 
Fig. 4a for the $1 \mu \mathrm{m}$ spherical indenter. As seen from this figure, cyclic loading and unloading of indenter in the post buckling state (Fig. 4a, Test B) produces hysteresis loops which suggest energy dissipation in each cycle. On the other hand, cyclic indentation at load levels well below the buckling load (Fig. 4a, Test A) show an increasing displacement at every load cycle - a behavior that is suggestive of delayed elasticity in the material due to van der Waals' interactions between the CNTs. Similar qualitative behaviors have also been noticed during axial compression of individual MWCNTs using AFM tips [21].
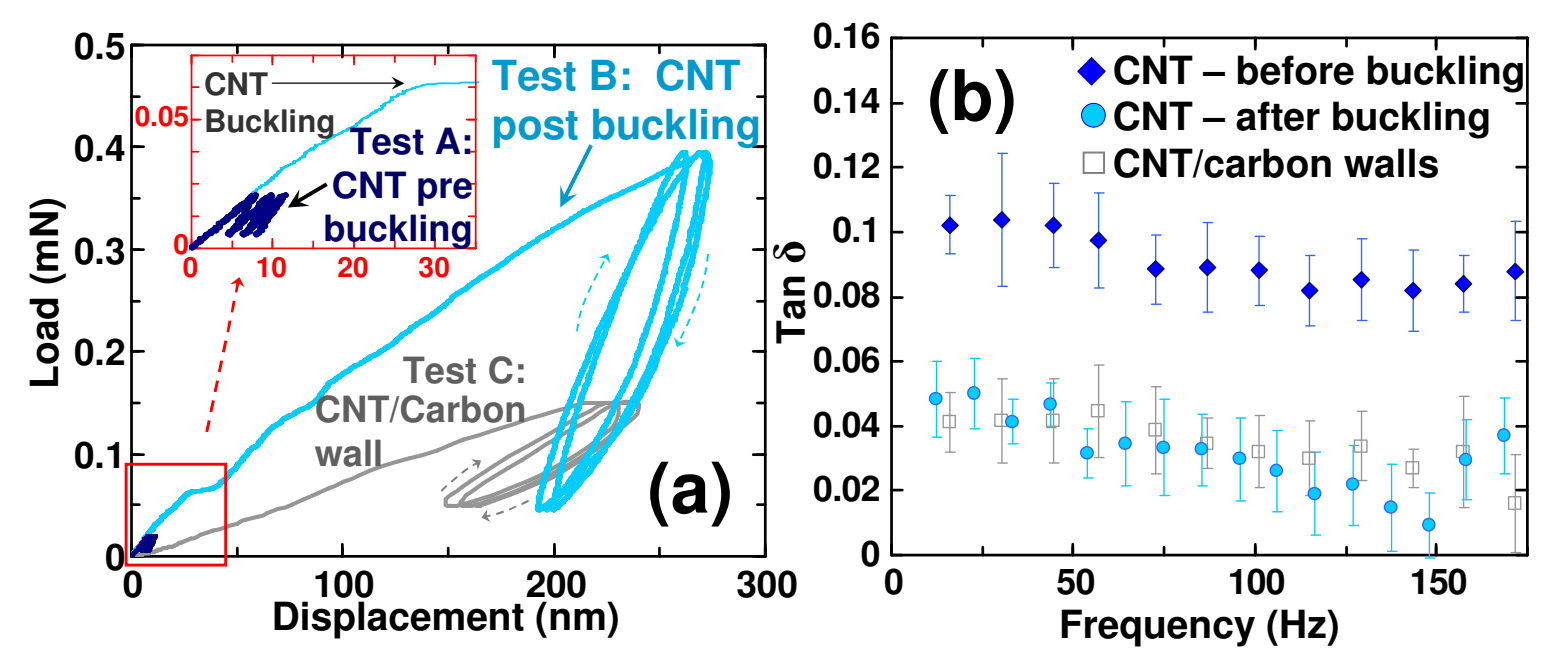

Figure 4. (a) Cyclic loading with a $1 \mu \mathrm{m}$ radius spherical indenter in a $1.3 \mu \mathrm{m}$ thick CNT brush grown by decomposition of $\mathrm{SiC}$ at $1800^{\circ} \mathrm{C}$ for 4 hours at load levels before buckling (Test $\mathrm{A}$ ), after buckling (Test B) and in a CNT/carbon wall mixture, grown by heating $\mathrm{SiC}$ wafer to $1900^{\circ} \mathrm{C}$ for 4 hours (Test C). Three cycles are shown in each test. (b) Viscoelastic indentation measurements showing significantly higher values of $\tan \delta$ at load levels before buckling in the CNT brush, than for CNTs after buckling or in a $\mathrm{CNT} /$ carbon wall mixture.

This damping behavior of the CNTs is also evident in the viscoelastic nanoindentation measurements shown in Fig. $4 \mathrm{~b}$ conducted using a $1 \mu \mathrm{m}$ spherical indenter. In this figure, the values for $\tan \delta$, defined as the loss modulus normalized by the storage modulus, serves as a measure of the energy loss in the material. As seen from Fig. 4b, oscillation of the indenter probe at a load level that is less than the critical buckling load results in a significant viscous response (higher values of $\tan \delta$, similar to those reported for individual MWCNTs [21]) from the CNT brush. On the other hand, indentations on the CNT brush at load levels higher than the buckling load do not show any appreciable viscoelasticity (low values of $\tan \delta$ which are comparable to the noise level of the machine). This is in contrast to the observations of Yap et al. [21], who 
noticed an increase in $\tan \delta$ in the initial post-buckled regime. The decrease in the $\tan \delta$ values in our case is most likely caused by the increased adhesive interactions between the individual CNTs in the brush when they are squeezed together after buckling. Note that the experiments in the work of Yap et al. were conducted on individual MWCNTs, where such adhesive interactions are non-existent. A similar response is seen in polymeric materials like rubber [46], where an increase in cross linking leads to a decrease of the viscoelastic response in the polymer.

Samples consisting of a mixture of carbon walls (graphitic structures growing normal to the surface) and CNTs, where a stronger interaction (cross-linking) between the graphite sheets is expected, also show a lower viscoelastic response, as evident in Fig. 4b. The observed viscoelastic behavior of the CNT brushes is expected to have significant implications in damping applications that utilize these materials. Note that the hysteresis loops in the CNT brushes (Fig. 4a, Test A) obtained at a rate of $0.01 \mathrm{~Hz}$ indicate energy losses on the order of $30 \%$. This implies that for large allowed displacements, motions over large sections of CNTs cause breaking of van der Waals' bonds, while the high frequency oscillations with small displacements show much lower losses (Fig. 4b). This is similar to polymer chains that have $\tan \delta$ peaks at different frequencies depending on what portion of the polymer chain is involved in the motion. Here, since the losses for hysteresis loop occur for displacements of $\sim 75 \mathrm{~nm}$, a relatively long portion of each CNT must be involved in the motion.

Note also that the mechanical properties of the CNT brushes are far superior to those of other graphitic structures such as vertically aligned carbon walls, which form during decomposition of $\mathrm{SiC}$ at a higher temperature of $1900{ }^{\circ} \mathrm{C}$ [34]. The modulus values calculated during cyclic indentations on a mixture of CNTs and carbon walls shown in Fig. 4a (Test C) is around $\sim 7 \mathrm{GPa}$, which is significantly lower compared to that of the CNT brushes. No buckling behavior is noted in these samples. However these specimens still show hysteresis loops upon cyclic loading ( Fig. 4a Test C), which indicates that van der Waals' forces still play an important role in these graphitic structures.

\section{Conclusion}

In summary, this study has shown that dense CNT brushes exhibit a mechanical behavior which is unique for this kind of material and distinctly different from that of graphite [47]. The ability of these dense non-catalytic CNTs to dissipate energy, while withstanding at least an 
order of magnitude higher loads compared to other nanotube brushes [5, 6] makes them highly promising for a variety of applications, especially in MEMS devices.

\section{Acknowledgements}

We thank Instrinsic for SiC wafers and Dr. V. Mochalin (Drexel University) and Dr. G. Yushin (currently with Georgia Tech.) for experimental help. Centralized Research Facility of the College of Engineering at Drexel University provided access to the microscopes and the MTS XP ${ }^{\circledR}$ System for nanoindentation used in this study. This work was supported by a grant from the US Department of Energy, office of Basic Energy Sciences (DE-FGOI-05ER05-01) S. P. also wishes to acknowledge the support from the Sigma Xi Grants-in-Aid of Research (GIAR) program and the 2007 Center for Integrated Nanotechnologies (CINT) user proposal grant at Los Alamos National Lab, Los Alamos, NM which provided access to the Hysitron TriboScope ${ }^{\circledR}$ used for the viscoelastic testing.

\section{References}

[1] Cao A, Dickrell PL, Sawyer WG, Ghasemi-Nejhad MN, Ajayan PM. Materials science: Super-compressible foamlike carbon nanotube films. Science 2005;310:1307.

[2] Gogotsi Y, editor Nanotubes and Nanofibers. Boca Raton, FL: CRC Press, 2006.

[3] Treacy MMJ, Ebbesen TW, Gibson JM. Exceptionally high Young's modulus observed for individual carbon nanotubes. Nature 1996;381:678.

[4] Min-Feng Y, Lourie O, Dyer MJ, Moloni K, Kelly TF, Ruoff RS. Strength and breaking mechanism of multiwalled carbon nanotubes under tensile load. Science 2000;287:637.

[5] Waters JF, Guduru PR, Jouzi M, Xu JM, Hanlon T, Suresh S. Shell buckling of individual multiwalled carbon nanotubes using nanoindentation. Appl. Phys. Lett. 2005;87:103109.

[6] Waters JF, Riester L, Jouzi M, Guduru PR, Xu JM. Buckling instabilities in multiwalled carbon nanotubes under uniaxial compression. Appl. Phys. Lett. 2004;85:1787.

[7] Tao T, Yang Z, Delzeit L, Kashani A, Meyyappan M, Majumdar A. Height independent compressive modulus of vertically aligned carbon nanotube arrays. Nano Letters 2008;8:511.

[8] Deck CP, Flowers J, McKee GSB, Vecchio K. Mechanical behavior of ultralong multiwalled carbon nanotube mats. Journal of Applied Physics 2007;101:23512.

[9] Yakobson BI, Brabec CJ, Bernholc J. Nanomechanics of carbon tubes: instabilities beyond linear response. Physical Review Letters 1996;76:2511.

[10] Wong EW, Sheehan PE, Lieber CM. Nanobeam mechanics: elasticity, strength and toughness of nanorods and nanotubes. Science 1997;277:1971.

[11] Wang CY, Ru CQ, Mioduchowski A. Applicability and Limitations of Simplified Elastic Shell Equations for Carbon Nanotubes. Journal of Applied Mechanics 2004;71:622.

[12] Wu J, Hwang KC, Huang Y. An atomistic-based finite-deformation shell theory for singlewall carbon nanotubes. Journal of the Mechanics and Physics of Solids 2008;56:279. 
[13] Xiao J, Jiang H, Khang DY, Wu J, Huang Y, Rogers JA. Mechanics of buckled carbon nanotubes on elastomeric substrates. Journal of Applied Physics 2008;104:033543.

[14] Zhang C-L, Shen H-S. Buckling and postbuckling analysis of single-walled carbon nanotubes in thermal environments via molecular dynamics simulation. Carbon 2006;44:2608.

[15] Mesarovic SD, McCarter CM, Bahr DF, Radhakrishnan H, Richards RF, Richards SD, McClain D, Jiao J. Mechanical behavior of a carbon nanotube turf. Scripta Materialia 2007;56:157.

[16] Minari-Jolandan M, Yu M-F. Reversible radial deformation up to the complete flattening of carbon nanotubes in nanoindentation. J. Appl. Phys. 2008;103:073516.

[17] Ler JGQ, Hao Y, Thong JTL. Effect of sidewall modification in the determination of friction coefficient of vertically aligned carbon nanotube films using friction force microscopy. Carbon 2007;45:2737.

[18] Olek M, Kempa K, Jurga S, Giersig M. Nanomechanical Properties of Silica-Coated Multiwall Carbon Nnaotubes-Poly(methyl methacrylate) Composites. Langmuir 2005;21:3146.

[19] Qi HJ, Teo KBK, Lau KKS, Boyce MC, Milne WI, Robertson J, Gleason KK. Determination of mechanical properties of carbon nanotubes and vertically aligned carbon nanotube forests using nanoindentation. J. Mech. Phys. Solids 2003;51:2213.

[20] Yap HW, Lakes RS, Carpick RW. Mechanical Instabilities of Individual Multiwalled Carbon Nanotubes under Cyclic Axial Compression. Nano Letters 2007;7:1149.

[21] Yap HW, Lakes RS, Carpick RW. Negative stiffness and enhanced damping of individual multiwalled carbon nanotubes. Physical Review B (Condensed Matter and Materials Physics) 2008;77:045423.

[22] Poncharal P, Wang ZL, Ugarte D, De Heer WA. Electrostatic deflections and electromechanical resonances of carbon nanotubes. Science 1999;283:1513.

[23] Pathak S, Kalidindi SR, Moser B, Klemenz C, Orlovskaya N. Analyzing indentation behavior of $\mathrm{LaGaO} 3$ single crystals using sharp indenters. Journal of the European Ceramic Society 2008;28:2039.

[24] Kalidindi SR, Pathak S. Determination of the effective zero-point and the extraction of spherical nanoindentation stress-strain curves. Acta Materialia 2008;56:3523.

[25] Pathak S, Kalidindi SR, Klemenz C, Orlovskaya N. Analyzing indentation stress-strain response of $\mathrm{LaGaO} 3$ single crystals using spherical indenters. Journal of the European Ceramic Society 2008;28:2213.

[26] Pathak S, Shaffer J, Kalidindi SR. Determination of an effective zero-point and extraction of indentation stress-strain curves without the continuous stiffness measurement signal. Scripta Materialia 2009;60:439.

[27] Asif SAS, Wahl KJ, Colton RJ, Warren OL. Quantitative imaging of nanoscale mechanical properties using hybrid nanoindentation and force modulation. Journal of Applied Physics 2001;90:1192.

[28] Herbert EG, Oliver WC, Pharr GM. Nanoindentation and the dynamic characterization of viscoelastic solids. Journal of Physics D: Applied Physics 2008;41:074021.

[29] Hongbing L, Gang H, Bo W, Mamedov A, Gupta S. Characterization of the linear viscoelastic behavior of single-wall carbon nanotube/polyelectrolyte multilayer nanocomposite film using nanoindentation. Thin Solid Films 2006;500:197.

[30] Li X, Gao H, Scrivens WA, Fei D, Xu X, Sutton MA, Reynolds AP, Myrick ML. Nanomechanical characterization of single-walled carbon nanotube reinforced epoxy composites. Nanotechnology 2004;15:1416. 
[31] Ajayan PM, Suhr J, Koratkar N. Utilizing interfaces in carbon nanotube reinforced polymer composites for structural damping. Journal of Materials Science 2006;41:7824.

[32] Kusunoki M, Rokkaku M, Suzuki T. Epitaxial carbon nanotube film self-organized by sublimation decomposition of silicon carbide. Applied Physics Letters 1997;71:2620.

[33] Kusunoki M, Suzuki T, Honjo C, Hirayama T, Shibata N. Selective synthesis of zigzag-type aligned carbon nanotubes on SiC (000-1) wafers. Chemical Physics Letters 2002;366:458.

[34] Cambaz ZG, Yushin G, Osswald S, Mochalin V, Gogotsi Y. Noncatalytic Synthesis of Carbon Nanotubes, Graphene and Graphite on SiC. Carbon 2008;46:841.

[35] Pharr GM, Strader JH, Oliver WC. Critical issues in making small-depth mechanical property measurements by nanoindentation with continuous stiffness measurement. Journal of Materials Research 2009;24:653.

[36] Jakes JE, Frihart CR, Beecher JF, Moon RJ, Stone DS. Experimental method to account for structural compliance in nanoindentation measurements. Journal of Materials Research 2008;23:1113.

[37] Hertz H. Miscellaneous Papers. New York: MacMillan and Co. Ltd., 1896.

[38] Love AEH. Boussinesq's problem for a rigid cone. J. Math 1939;10:161.

[39] Oliver WC, Pharr GM. Improved technique for determining hardness and elastic modulus using load and displacement sensing indentation experiments. Journal of Materials Research 1992; 7:1564.

[40] Oliver WC, Pharr GM. Measurement of hardness and elastic modulus by instrumented indentation: Advances in understanding and refinements to methodology. Journal of Materials Research 2004;19:3.

[41] Pathak S, Stojakovic D, Kalidindi SR. Measurement of the Local Mechanical Properties in Polycrystalline Samples Using Spherical Nano-Indentation and Orientation Imaging Microscopy. Acta Materialia 2009;57:3020.

[42] Pathak S, Stojakovic D, Doherty R, Kalidindi SR. Importance of Surface Preparation on the Nano-Indentation Stress-Strain Curves Measured in Metals. Journal of Materials Research Focus Issue on Nanoindentation March 2009;24:1127.

[43] Liu L, Cao G, Chen X. Mechanisms of Nanoindentation on Multiwalled Carbon Nanotube and Nanotube Cluster. Journal of Nanomaterials 2008;2008.

[44] Yamada T, Namai T, Hata K, Futaba DN, Mizuno K, Fan J, Yudasaka M, Yumura M, Iijima S. Size-selective growth of double-walled carbon nanotube forests from engineered iron catalysts. Nature Nanotechnology 2006;1:131.

[45] Gere JM, Timoshenko SP. Mechanics of Materials: PWS Publishers, Boston, MA, USA, 1984.

[46] Ward IM, Sweeney J. An Introduction to the Mechanical Properties of Solid Polymers: Wiley, 2004.

[47] Barsoum MW, Murugaiah A, Kalidindi SR, Zhen T, Gogotsi Y. Kink bands, nonlinear elasticity and nanoindentations in graphite. Carbon 2004;42:1435. 